\title{
埋嵌在超薄 $\mathrm{Al}_{2} \mathrm{O}_{3}$ 薄膜中的 $\mathrm{Ni}$ 纳米颗粒在生长过程 中的应变场分布
}

张求龙 ${ }^{(1)}$, 江子雄 ${ }^{(1)}$, 袁彩雷 $^{(1)}$, 张振荣 ${ }^{(2)}$

(1) 江西师范大学物理与通信电子学院, 南昌 330022 ;

(2) 广西大学计算机与电子信息学院, 南宁 530004

*联系人, E-mail: clyuan@jxnu.edu.cn

收稿日期: 2012-03-29; 接受日期: 2012-05-25; 网络出版日期: 2012-06-18

国家自然科学基金(批准号: 11004087, 11164008)和江西省自然科学基金(编号: 2009GQW0007)资助项目

摘要对于埋嵌在介电材料中的纳米颗粒, 在其生长过程中总是不可避免的伴随着应变场的产生, 而 这种应变场会的分布对纳米颗粒的物理性能产生重要的影响. 在本文中用脉冲激光沉积技术和快速退火 成功地制备了埋嵌在超薄 $\mathrm{Al}_{2} \mathrm{O}_{3}$ 薄膜中的 $\mathrm{Ni}$ 纳米颗粒. 利用高分辨透射电子显微镜观察到立方形 $\mathrm{Ni}$ 纳 米颗粒埋嵌在超薄 $\mathrm{Al}_{2} \mathrm{O}_{3}$ 薄膜中. 用有限元算法系统地模拟了 $\mathrm{Ni}$ 纳米颗粒生长过程中的应变场分布, 研 究工作发现, 随着 $\mathrm{Ni}$ 纳米颗粒的生长, $\mathrm{Ni}$ 纳米颗粒受到了非均匀偏应变作用逐渐增强, 而且变得越来越 不均匀, 这种非均匀的偏应变作用对于纳米颗粒的微观结构有很大的影响. 可以通过调节 $\mathrm{Ni}$ 纳米颗粒生 长过程中的应变场来实现对 $\mathrm{Ni}$ 纳米颗粒界面态的调控, 从而进一步优化 $\mathrm{Ni}$ 纳米颗粒的物理性能. 系统 地研究磁性 $\mathrm{Ni}$ 纳米材料的应变场分布，对有效的调控其物理化学性能有着非常重大的意义.

关键词磁性纳米颗粒, 应变, 透射电子显微镜

PACS: 75.75.Cd, 77.75.Jn, 77.80.bn

doi: $10.1360 / 132012-237$

最近, 人们发现埋嵌在介电材料中的纳米颗粒, 在纳米颗粒的生长过程中总是不可避免的伴随着应 变的产生 ${ }^{[1-3]}$, 应变作用可以有效地改变原子之间、 分子之间的距离, 从而改变原子之间和分子之间的 相互作用, 调节带隙宽度, 能够获得与常压下完全不 同的新现象、新规律是深入研究材料的结构、性质和 获得新物质、发现新结构的重要手段 ${ }^{[4,5]}$. 同时, 我们
的研究工作发现, 不同尺寸的纳米颗粒在生长过程 中, 受到应变场的分布有很大的不同, 这种应变场的 不同对于纳米颗粒的微观结构和形貌都有很大影

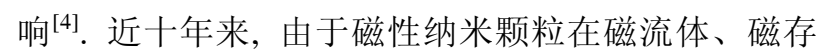
储媒介等方面有着很广泛的应用 ${ }^{[6,7]}$. 而随着存储设 备的小型化和高密度化, 存储器件的体积越来越小, 存储量越来越大, 对于埋嵌型的存储介质, 要求埋嵌

引用格式: 张求龙, 江子雄, 袁彩雷, 等. 埋嵌在超薄 $\mathrm{Al}_{2} \mathrm{O}_{3}$ 薄膜中的 $\mathrm{Ni}$ 纳米颗粒在生长过程中的应变场分布. 中国科学: 物理学 力学 天文学, 2012, $42:$ $711-715$

Zhang Q L, Jiang Z X, Yuan C L, et al. Strain distribution of Ni nanoparticles embedded in $\mathrm{Al}_{2} \mathrm{O}_{3}$ ultrathin film (in Chinese). Sci Sin-Phys Mech Astron, 2012, 42: 711-715, doi: 10.1360/132012-237 
纳米材料的薄膜必须越做越薄 ${ }^{[8-13]}$. 而在薄膜中, 由 于纳米颗粒生长产生的应变对纳米颗粒和薄膜的物 理性质有更加显著的影响. 另外, 纳米颗粒处在薄膜 中的位置不同, 其生长过程中所受到的应变的情况 存在很大的差异 ${ }^{[14]}$. 但是到目前为止, 对磁性纳米颗 粒在超薄薄膜中的生长过程中的应变场分布还缺乏 比较系统的研究. 因此, 成功制备埋嵌在超薄 $\mathrm{Al}_{2} \mathrm{O}_{3}$ 薄膜中的磁性纳米颗粒, 并系统地认识 $\mathrm{Ni}$ 纳米颗粒 在生长过程中的应变场分布, 对于磁性 $\mathrm{Ni}$ 纳米材料 的应用前景有非常重要的意义. 单击此处键入前言 内容.

\section{1 实验部分}

我们利用脉冲激光沉积技术和快速退火成功制 备了埋嵌在非晶 $\mathrm{Al}_{2} \mathrm{O}_{3}$ 薄膜中的磁性 $\mathrm{Ni}$ 纳米颗粒. 准 分子脉冲激光的波长为 $248 \mathrm{~nm}$, 频率为 $5 \mathrm{~Hz}$. 靶是 由一个直径为 $25 \mathrm{~mm}$ 的圆形的高纯度 $(99.9 \%)$ 的 $\mathrm{Al}_{2} \mathrm{O}_{3}$ 靶材和一块长 $3 \mathrm{~mm}$ 的方形 $\mathrm{Ni}$ 靶材组成, 保持 $\mathrm{Al}_{2} \mathrm{O}_{3}$ 靶材和 $\mathrm{Ni}$ 靶材始终是物理性的黏结, 而非化学 性的黏结. 在沉积过程中, 我们利用一束准分子脉冲 激光烧蚀固体靶, $\mathrm{Al}_{2} \mathrm{O}_{3}$ 和 $\mathrm{Ni}$ 组成的靶材缓慢的围绕 中心轴旋转, 激光光束交替地烧蚀靶材的两种材料. $\mathrm{P}$ 型(100)硅祄底先用 $\mathrm{SC} 1\left(\mathrm{NH}_{4} \mathrm{OH}: \mathrm{H}_{2} \mathrm{O}_{2}: \mathrm{H}_{2} \mathrm{O}=1: 1: 5\right)$ 和 $\mathrm{SC} 2\left(\mathrm{HCl}: \mathrm{H}_{2} \mathrm{O}_{2}: \mathrm{H}_{2} \mathrm{O}=1: 1: 5\right)$ 清洁, 然后浸入 $1 \%$ 的 $\mathrm{HF}$ 溶液以去除表面氧化层. 在整个沉积的过程中真 空室的真空度为 $7 \times 10^{-8}$ Torr $\left(1\right.$ Torr $\left.=1.333 \times 10^{2} \mathrm{~Pa}\right)$, 同时保持硅祄底的温度为室温. 沉积下来的样品在 氮气中 $600^{\circ} \mathrm{C}$ 快速退火 $120 \mathrm{~s}$. 使用 $2010 \mathrm{JEOL}$ 高分辨 透射电子显微镜(HRTEM) 观察这些样品的微观结构.

\section{2 模拟与讨论}

图 1 是经过 $600^{\circ} \mathrm{C}$ 快速退火样品的高分辨透射电 子显微镜图像. 图 1 中显示了一个立方形 $\mathrm{Ni}$ 纳米颗 粒埋嵌在超薄非晶 $\mathrm{Al}_{2} \mathrm{O}_{3}$ 薄膜中. $\mathrm{Ni}$ 纳米颗粒的生长 位置接近非晶 $\mathrm{Al}_{2} \mathrm{O}_{3}$ 薄膜的上表面. $\mathrm{Ni}$ 纳米颗粒的尺 寸约为 $10 \mathrm{~nm}$ 左右, 薄膜的厚度 $12 \mathrm{~nm}$ 左右. $\mathrm{Ni}$ 纳米 颗粒形成的机理如下: 随着退火温度的升高, $\mathrm{Ni}$ 原子 形成核的几率也提高, 纳米粒子的密度也会变大; 只 要表面能足够, 会形成一些 $\mathrm{Ni}$ 核, 而在其周围的 $\mathrm{Ni}$ 原子会通过表面扩散依附到已形成的 Ni 核上，团聚

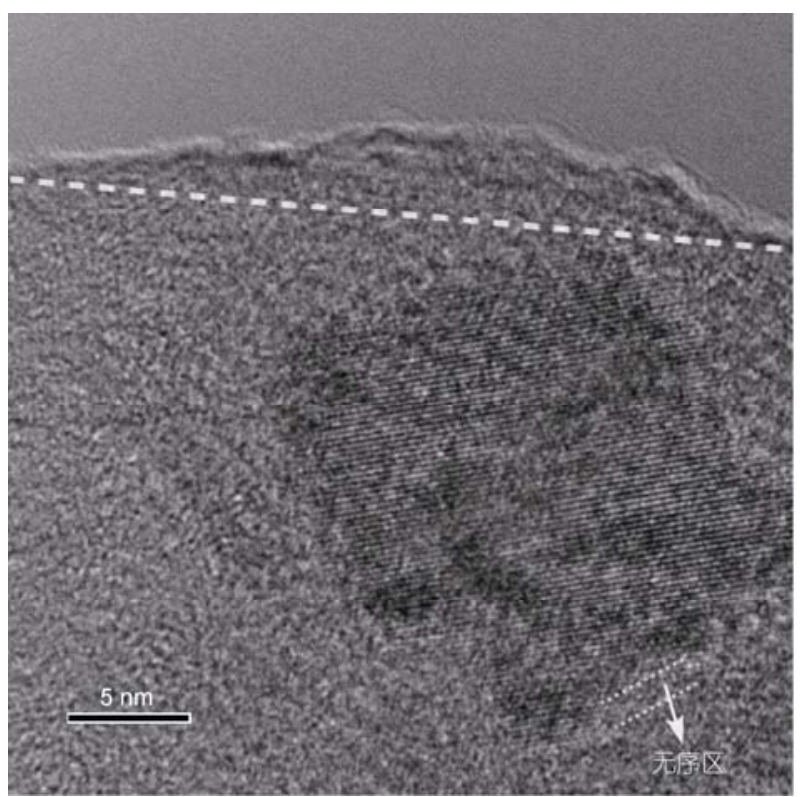

图 1 经过 $600^{\circ} \mathrm{C}$ 快速退火样品的高分辨电子显微镜图像 Figure 1 HRTEM image of the sample after annealing at $600^{\circ} \mathrm{C}$.

成一个更大的 $\mathrm{Ni}$ 纳米颗粒 ${ }^{[15]}$. 图 1 还显示 $\mathrm{Ni}$ 纳米颗 粒上表面上方的 $\mathrm{Al}_{2} \mathrm{O}_{3}$ 薄膜向上拱起, 同时, $\mathrm{Ni}$ 纳米 颗粒上表面和非晶 $\mathrm{Al}_{2} \mathrm{O}_{3}$ 的界面处, 晶格条纹比较清 晰; 而在 $\mathrm{Ni}$ 纳米颗粒的下表面与非晶 $\mathrm{Al}_{2} \mathrm{O}_{3}$ 的界面处, 晶格条纹不是很清晰, 部分区域出现了无序区.

$\mathrm{Ni}$ 纳米颗粒埋嵌在超薄薄膜中产生应变的模型 基于以下假设：一个立方形的各向同性的线弹性的 纳米颗粒置于一个无限大的各向同性的线弹性的薄 膜中, 纳米颗粒是埋嵌在薄膜中的. 假设纳米颗粒被 放在薄膜的一个非常小的空腔中, 由于周围薄膜材 料的原子不能迅速的适应纳米颗粒在生长过程中的 体积的变化, 因而导致了纳米颗粒受到了周围薄膜 材料的压缩应变. 我们用有限元算法(ANSYS 软件) 定性地模拟了二维的埋嵌在超薄 $\mathrm{Al}_{2} \mathrm{O}_{3}$ 薄膜中的 $\mathrm{Ni}$ 纳米颗粒生长过程中的应变场强度的分布 ${ }^{[16-18]}$. 在 模拟中, $\mathrm{Ni}$ 和 $\mathrm{Al}_{2} \mathrm{O}_{3}$ 的杨氏模量分别是 207 和 $360 \mathrm{GPa}$, 泊松比分别是 0.291 和 0.24 . 在有限元计算中, 假设 方形的 $\mathrm{Ni}$ 纳米颗粒的位置在超薄 $\mathrm{Al}_{2} \mathrm{O}_{3}$ 薄膜的中心, $\mathrm{Ni}$ 纳米颗粒和 $\mathrm{Al}_{2} \mathrm{O}_{3}$ 薄膜的交界处是埋嵌的.

图 2 是尺寸为 $2.5,5,7.5$ 和 $10 \mathrm{~nm}$ 的 $\mathrm{Ni}$ 纳米颗粒 埋嵌在超薄 $\mathrm{Al}_{2} \mathrm{O}_{3}$ 薄膜中的 $X-Y$ 剖面的应力场分布图, 图 3 是尺寸为 $2.5,5,7.5$ 和 $10 \mathrm{~nm}$ 的 $\mathrm{Ni}$ 纳米颗粒的 $X-Y$ 剖面的应变强度图. 显然 $\mathrm{Ni}$ 纳米颗粒受到周围 $\mathrm{Al}_{2} \mathrm{O}_{3}$ 薄膜的压缩应变. 从图 2 和 3 可以看出, $\mathrm{Ni}$ 纳米 


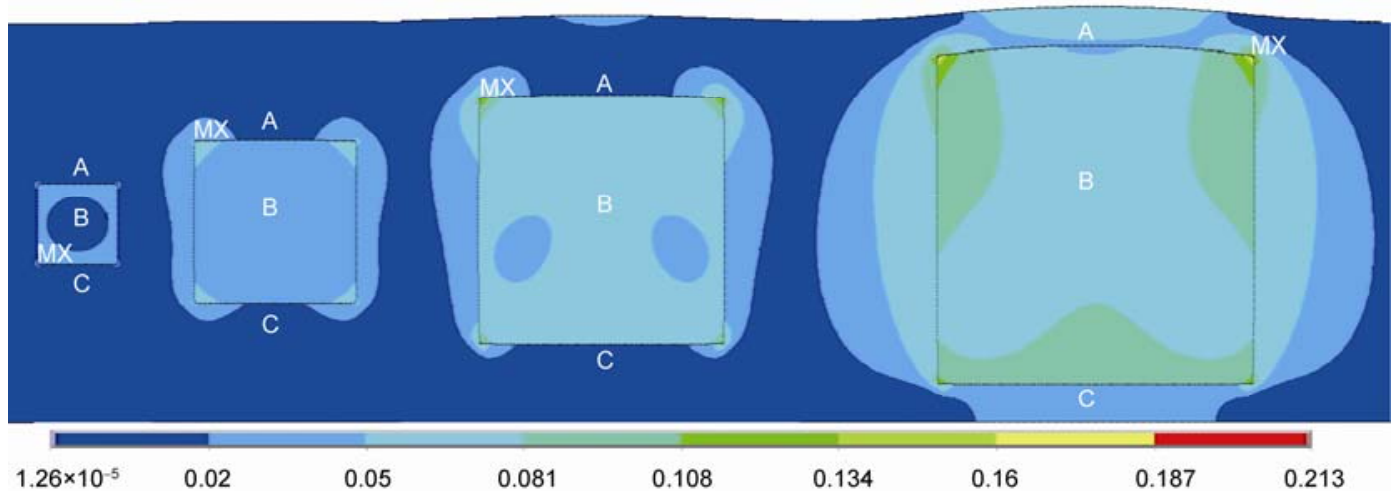

图 2 (网络版彩图) 尺寸分别为 $2.5,5,7.5$ 和 $10 \mathrm{~nm}$ 的 $\mathrm{Ni}$ 纳米颗粒的 $X-Y$ 剖面的应变场分布

Figure 2 (Color online) $X-Y$ plane strain distribution of Ni nanoparticle with the size of 2.5, 5, 7.5 and $10 \mathrm{~nm}$.
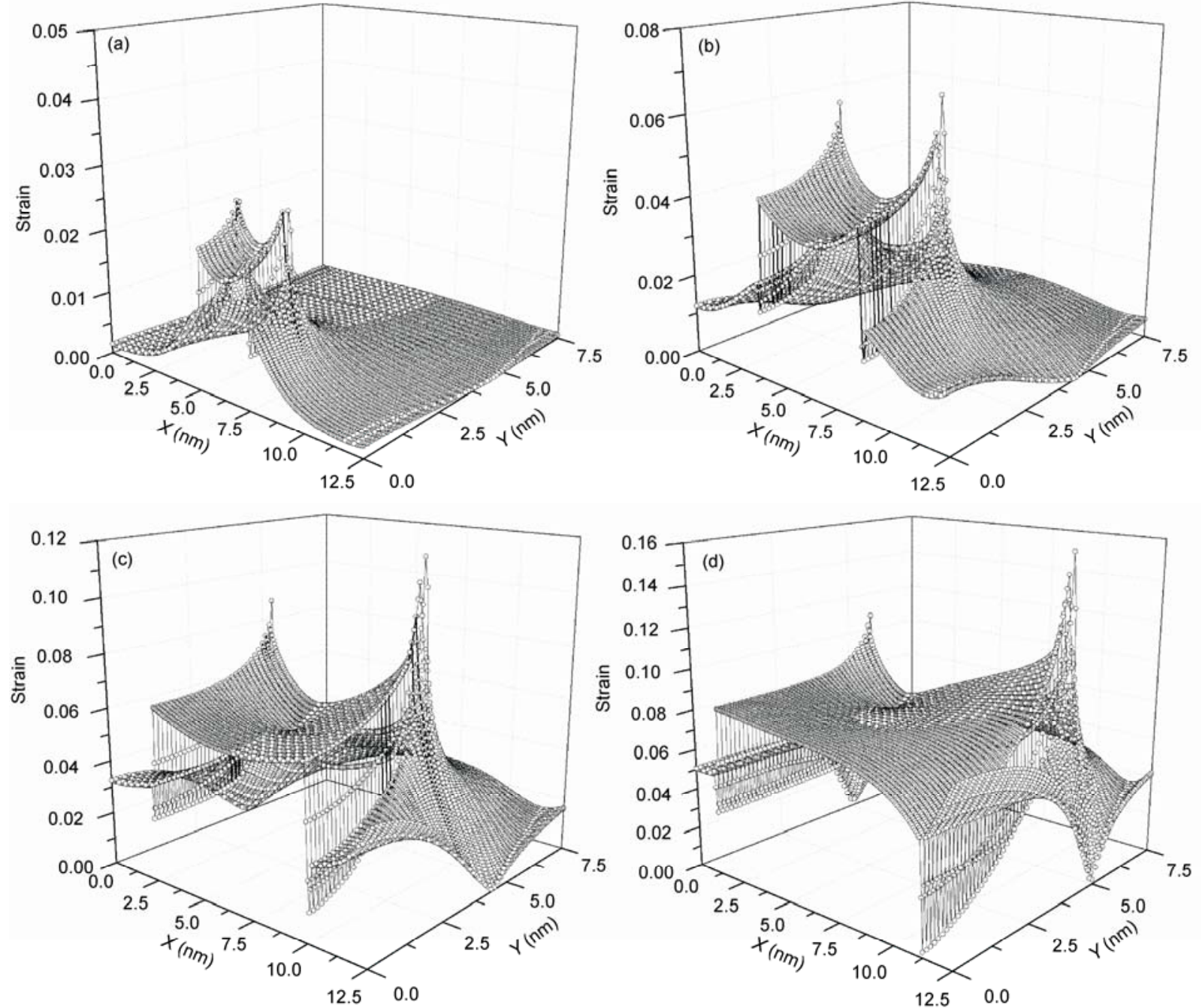

图 $3 \mathrm{Ni}$ 纳米颗粒的 $X-Y$ 剖面的应变强度图

(a) $2.5 \mathrm{~nm}$; (b) $5 \mathrm{~nm}$; (c) $7.5 \mathrm{~nm}$; (d) $10 \mathrm{~nm}$

Figure $3 X$-Y plane strain profile of Ni nanoparticle with the size. (a) $2.5 \mathrm{~nm}$; (b) $5 \mathrm{~nm}$; (c) $7.5 \mathrm{~nm}$; (d) $10 \mathrm{~nm}$. 
颗粒受到了非均匀偏应变作用. 随着 $\mathrm{Ni}$ 纳米颗粒在 超薄 $\mathrm{Al}_{2} \mathrm{O}_{3}$ 薄膜中的生长, 它所受的周围薄膜材料的 非均匀偏应变也逐渐增强. 当 $\mathrm{Ni}$ 纳米颗粒的尺寸较 小(如图 2 中 2.5 和 $5 \mathrm{~nm}$ 的情况)时, $\mathrm{Ni}$ 纳米颗粒受到 的薄膜材料的非均匀偏应变作用不是很强; 而当 $\mathrm{Ni}$ 纳米颗粒的尺寸长大(如图 2 中 7.5 和 $10 \mathrm{~nm}$ 的情 况)后, $\mathrm{Ni}$ 纳米颗粒受到的薄膜材料的压缩应变呈现 出上下不对称分布. 特别是当 $\mathrm{Ni}$ 纳米颗粒接近薄 膜的表面时, 其受到的偏应变的分布变得极其的不 均匀.

图 4 为对应于图 2 中样品 $1(2.5 \mathrm{~nm}), 2(5 \mathrm{~nm})$, $3(7.5 \mathrm{~nm}), 4(10 \mathrm{~nm})$ 的 A, B, C 点的应变变化曲线, A 点位于 $\mathrm{Ni}$ 纳米颗粒的上表面的面心位置, $\mathrm{B}$ 点位于 $\mathrm{Ni}$ 纳米颗粒的体心位置, $\mathrm{C}$ 点位于 $\mathrm{Ni}$ 纳米颗粒的下 表面的面心位置. 图 4 表明, 2.5 和 $5 \mathrm{~nm}$ 时, $\mathrm{Ni}$ 纳米 颗粒的上表面的面心位置和下表面的面心位置所受 到的应变相同, 体心位置受到的应变略小于上下表 面中心位置受到的应变; $7.5 \mathrm{~nm}$ 时, $\mathrm{Ni}$ 纳米颗粒整体 受到的偏应变作用强大增大了, 而上表面的面心位 置受到的偏应变相对于下表面的面心位置受到的偏 应变应变减小了, $10 \mathrm{~nm}$ 时这种应变的减小程度就更 加明显了. 这是由于埋嵌在超薄 $\mathrm{Al}_{2} \mathrm{O}_{3}$ 薄膜中的 $\mathrm{Ni}$ 纳米颗粒在生长过程中会受到非均匀偏应变作用, 随着 $\mathrm{Ni}$ 纳米颗粒的生长, 它所受的非均匀偏应变作 用逐渐增强, 然而当 $\mathrm{Ni}$ 纳米颗粒接近薄膜的上表面 时, $\mathrm{Ni}$ 纳米颗粒受到薄膜表面偏应变作用, 由于相互 作用, 薄膜表面也会受到来自 $\mathrm{Ni}$ 纳米颗粒的应变, 所以薄膜上表面会因为受到应变而发生形变, 正如 上文图 1 中显示的 $\mathrm{Ni}$ 纳米颗粒上表面上方的 $\mathrm{Al}_{2} \mathrm{O}_{3}$ 薄膜向上拱起, 从而很大程度上释缓了 $\mathrm{Ni}$ 纳米颗粒 的上表面所受到的应变, 因而在 $\mathrm{Ni}$ 纳米颗粒上表面 和 $\mathrm{Al}_{2} \mathrm{O}_{3}$ 薄膜的界面处晶格条纹比较清晰; 而 $\mathrm{Ni}$ 纳米 颗粒的下表面由于受到的应变较大, 所以会在部分

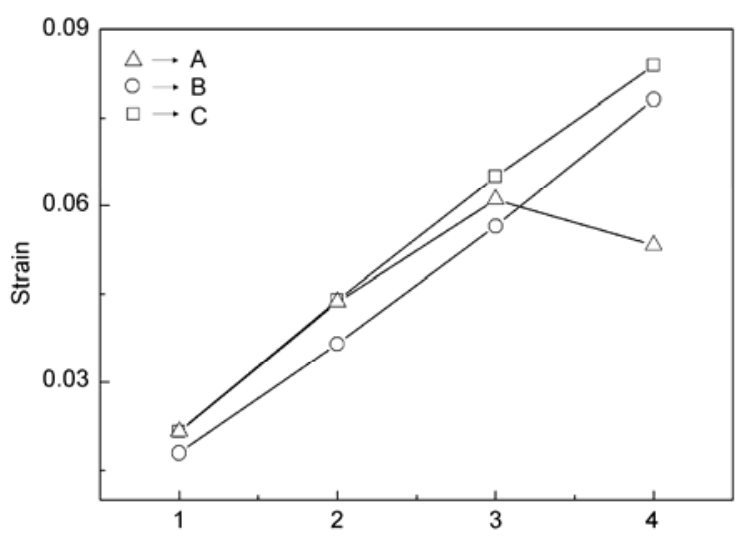

图 4 对应于图 2 中样品 $1(2.5 \mathrm{~nm}), 2(5 \mathrm{~nm}), 3(7.5 \mathrm{~nm}), 4(10$ $\mathrm{nm})$ 的 $\mathrm{A}, \mathrm{B}, \mathrm{C}$ 点的应变变化曲线

Figure 4 Strain intensity of the point of $\mathrm{A}, \mathrm{B}$ and $\mathrm{C}$ in the sample $1(2.5 \mathrm{~nm}), 2(5 \mathrm{~nm}), 3(7.5 \mathrm{~nm})$ and $4(10 \mathrm{~nm})$, respectively.

区域出现无序区, 从而释缓掉一部分应变. 这也造成 了 $\mathrm{Ni}$ 纳米颗粒受到了更加不均匀的偏应变作用. 这 种存在的非均匀偏应变作用会导致 $\mathrm{Ni}$ 纳米颗粒物理 性质发生变化, 因此, 研究纳米颗粒生长过程中的应 变场分布对于纳米颗粒的应用有非常重要的意义 ${ }^{[14]}$.

\section{3 结论}

我们用脉冲激光沉积和快速退火技术成功制备 了埋嵌在超薄 $\mathrm{Al}_{2} \mathrm{O}_{3}$ 薄膜上的 $\mathrm{Ni}$ 纳米颗粒. 用有限元 算法研究 $\mathrm{Ni}$ 纳米颗粒在超薄 $\mathrm{Al}_{2} \mathrm{O}_{3}$ 薄膜中的生长过 程中的应变场分布, 发现在 $\mathrm{Ni}$ 纳米颗粒的生长过程 中, $\mathrm{Ni}$ 纳米颗粒受到了非均匀偏应变作用. 随着 $\mathrm{Ni}$ 纳米颗粒的尺寸变大, 它所受的薄膜的非均匀偏应 变也逐渐增强，同时其受到的应变场的分布变得更 加不均匀. 这种极其不均匀的应变对于纳米颗粒的 微观结构有很大的影响. 因此, 系统地研究磁性 $\mathrm{Ni}$ 纳米颗粒的在超薄薄膜中的应变场分布, 对有效的 调控其物理化学性能有着非常重大的意义.

\section{参考文献}

1 Choi W K, Ng V, Ng S P, et al. Raman characterization of germanium nanocrystals in amorphous silicon oxide films synthesized by rapid thermal annealing. J Appl Phys, 1999, 86: 1398-1403

2 Wellner A, Paillard V, Bonafos C, et al. Stress measurements of germanium nanocrystals embedded in silicon oxide. J Appl Phys, 2003, 94: 5639-5642

3 Chew H G, Zheng F, Choi W K, et al. Influence of reductant and germanium concentration on the growth and stress development of germanium nanocrystals in silicon oxide matrix. Nanotechnology, 2007, 18: 065302 
4 Yuan C L, Liu Q, Xu B. Strain-induced structural phase transition of Si nanoparticles. J Phys Chem C, 2011, 115(33): 16374-16377

5 Yuan C L, Xu B, Lei W. Strain-induced direct band gap $\mathrm{LaAlO}_{3}$ nanocrystals. Mater Lett, 2012, 68(1): 392-394

6 Pileni M P. Magnetic fluids: Fabrication, magnetic properties, and organization of nanocrystals. Adv Funct Mater, 2001, 11(5): 323-336

7 Sun S H, Murray C B, Weller D, et al. Monodisperse FePt nanoparticles and ferromagnetic FePt nanocrystal superlattices. Science, 2000, 287(5460): 1989-1992

8 Eigler D M, Schweizer E K. Positioning single atoms with a scanning tunneling microscope. Nature, 1990, 344(6266): 524-526

9 Binnig G, Despont M, Drechsler U, et al. Ultrahigh-density atomic force microscopy data storage with erase capability. Appl Phys Lett, 1999, 74(9): 1329-1331

10 Lutwyche M I, Despont M, Drechsler U, et al. Highly parallel data storage system based on scanning probe arrays. Appl Phys Lett, 2000, 77(20): 3299-3301

11 Cumpston B H, Ananthavel S P, Barlow S, et al. Two-photon polymerization initiators for three-dimensional optical data storage and microfabrication. Nature, 1999, 398(6722): 51-54

12 Hosaka S, Shintani T, Miyamoto M, et al. Scanning near-field optical microscope with a laser diode and nanometer-sized bit recording. J Appl Phys, 1996, 79(10): 8082-8086

13 Chou S Y, Krauss P R, Renstrom P J. Imprint lithography with 25-nanometer resolution. Science, 1996, 272(5258): 85-87

14 Yuan C L, Lei W. Photoluminescence of $\mathrm{Al}_{2} \mathrm{O}_{3}$ nanocrystals induced by compressive stress. Physica E, 2010, 42(5): 1687-1690

15 Yuan C L, Lee P S, Ye S L. Formation, photoluminescence and charge storage characteristics of Au nanocrystals embedded in amorphous $\mathrm{Al}_{2} \mathrm{O}_{3}$ matrix. Europhys Lett, 2007, 80(6): 67003

16 Benabbas T, Androussi Y, Lefebvre A. A finite-element study of strain fields in vertically aligned InAs islands in GaAs. J Appl Phys, 1999, 86: 1945-1950

17 Pei Q X, Lu C, Wang Y Y. Effect of elastic anisotropy on the elastic fields and vertical alignment of quantum dots. J Appl Phys, 2003, 93: 1487-1492

18 Shin H, Lee W, Yoo Y H. Comparison of strain fields in truncated and un-truncated quantum dots in stacked InAs/GaAs nanostructures with varying stacking periods. J Phys Condens Matter, 2003, 15(22): 3689-3699

\title{
Strain distribution of Ni nanoparticles embedded in $\mathrm{Al}_{2} \mathrm{O}_{3}$ ultrathin film
}

\author{
ZHANG QiuLong $^{1}$, JIANG ZiXiong ${ }^{1}$, YUAN CaiLei ${ }^{1 *} \&$ ZHANG ZhenRong ${ }^{2}$ \\ ${ }^{1}$ School of Physics, Communication and Electronics, Jiangxi Normal University, Nanchang 330022, China; \\ ${ }^{2}$ School of Computer, Electronics and Information, Guangxi University, Nanning 530004, China
}

Nucleation and growth lead to substantial strain in nanoparticles embedded in a host dielectrics matrix. The distribution of strain field plays an important role in the physical properties of nanoparticles. Ni nanoparticles embedded in the amorphous $\mathrm{Al}_{2} \mathrm{O}_{3}$ ultrathin films were fabricated by using pulsed laser deposition and rapid thermal annealing. The results from high-resolution transmission electron microscope also revealed that the complete isolation of $\mathrm{Ni}$ nanoparticles with cubic shape embedded in ultrathin amorphous $\mathrm{Al}_{2} \mathrm{O}_{3}$ matrix. The growth strain of $\mathrm{Ni}$ nanoparticle embedded in the $\mathrm{Al}_{2} \mathrm{O}_{3}$ matrix was investigated. Finite element calculations clearly indicate that the $\mathrm{Ni}$ nanoparticle incurs a net deviatoric strain. With the growth of Ni nanoparticle, the larger Ni nanoparticles incur stronger net deviatoric strain and the strain become more and more nonuniform, which will have much influence on the structure and morphology of Ni nanoparticles. Strain engineering is an effective tool for tailoring the properties of Ni nanoparticles.

magnetic nanoparticles, strain, transmission electron microscope

PACS: 75.75.Cd, 77.75.Jn, 77.80.bn

doi: $10.1360 / 132012-237$ 